\title{
Molecular organization of the decapentaplegic gene in Drosophila melanogaster
}

\author{
R. Daniel St. Johnston, ${ }^{1}$ F. Michael Hoffmann, ${ }^{2}$ Ronald K. Blackman, ${ }^{3}$ Daniel Segal, ${ }^{4}$ \\ Raymond Grimaila, ${ }^{5}$ Richard W. Padgett, Holly A. Irick, ${ }^{6}$ and William M. Gelbart \\ Department of Cellular and Developmental Biology, Harvard University, Cambridge, Massachusetts 02138-2097 USA
}

\begin{abstract}
The decapentaplegic (dpp) locus of Drosophila melanogaster is a $>55 \mathrm{~kb}$ genetic unit required for proper pattern formation during the embryonic and imaginal development of the organism. We have proposed that these morphogenetic functions result from the action of a secreted transforming growth factor- $\beta$ (TGF- $\beta$ )-related protein product encoded by $d p p$. In this paper we localize 60 mutations on the molecular map of $d p p$. The positions of these mutations cluster according to phenotypic class, identifying the locations of specific $d p p$ functions. By Northern and cDNA analysis, we characterize five overlapping $d p p$ transcripts. On the basis of the locations of the overlaps relative to a previously sequenced cDNA, it is likely that these transcripts all encode similar or identical polypeptides. We propose that the bulk of $d p p$ DNA consists of extensive arrays of cisregulatory information. The large $(>25-\mathrm{kb}) 3^{\prime}$ cis-regulatory region represents a novel feature of $d p p$ gene organization.
\end{abstract}

[Key Words: Drosophila; $d p p$ gene; imaginal disks; TGF- $\beta$ family; pattern formation]

Received February 14, 1990; revised version accepted April 16, 1990.

The embryonic body plan of Drosophila melanogaster is established within several hours of fertilization, as a result of the spatially restricted activation of zygotic genes by maternal signals. Two classes of zygotic genes are involved in the earliest steps of dorsal-ventral determination (for review, see Anderson 1987). In the first class, twist and snail mutations both produce a weak dorsalization, in which the most ventral pattern element, the mesoderm, is absent (Nüsslein-Volhard et al. 1984). twist is first expressed at the blastoderm stage, in the presumptive mesodermal cells (Thisse et al. 1987). The snail sequence contains $\mathrm{Zn}$-binding fingers, whereas twist encodes a myc-related protein that is localized to the nucleus, suggesting that both proteins bind to DNA (Boulay et al. 1987; Thisse et al. 1988; Murre et al. 1989). In the second class, mutations in at least seven genes [zerknüllt (zen), decapentaplegic (dpp), tolloid, short gastrulation, twisted gastrulation, screw, shrew] produce a partially ventralized phenotype, in which the dorsal-most structures of the embryo, the amnioserosa and the dorsal epidermis, are reduced or absent (Jürgens et al. 1984; Nüsslein-Volhard et al. 1984; Wakimoto et

Present addresses: 'Max Planck Institut für Entwicklungsbiologie, Abteilung III, D-7400 Tübingen, West Germany; ${ }^{2}$ Department of Oncology, McArdle Laboratory, University of Wisconsin, Madison, Wisconsin 53706 USA; $^{3}$ Cell and Structural Biology, University of Illinois, Urbana, Illinois 61801 USA; ${ }^{4}$ Department of Microbiology, Tel-Aviv University, Tel-Aviv 69978 Israel; ${ }^{5}$ Repligen Corp., Cambridge, Massachusetts 02139 USA; ${ }^{7}$ Department of Biology, Indiana University, Bloomington, Indiana 47405 USA. al. 1984; Zusman and Wieschaus 1985; Irish and Gelbart 1987; Zusman et al. 1988). The zen gene, which mutates to produce a weakly ventralized phenotype, is expressed dorsally in early embryos (Doyle et al. 1986). Like twist and snail, zen encodes a nuclear homeo domain-containing protein that probably binds DNA (Rushlow et al. 1987).

The most extreme zygotic ventralizing phenotypes are elicited by null mutations at $d p p$ ( $d p p^{\text {Hin }}$ alleles). These embryos fail to complete germ-band extension and develop to first-instar larvae with no detectable dorsal epidermal derivatives (Irish and Gelbart 1987). Instead, the dorsal cells follow a more ventral pathway of development and differentiation to secrete ventral and lateral cuticle with the characteristic denticle belts. Consistent with its early function in dorsal determination, $d p p$ is expressed first in the dorsal region of early syncytial blastoderm embryos and, after gastrulation begins, becomes restricted to the dorsal ectoderm (St. Johnston and Gelbart 1987). Subsequent expression of $d p p$ in the dorsal and lateral ectoderm, and in the visceral mesoderm and other internal tissues suggests that $d p p$ may provide additional embryonic functions.

On the basis of the sequence of two cDNAs (Padgett et al. 1987), $d p p$ is predicted to encode a polypeptide homologous to a family of secreted factors known in mammals to include transforming growth factor- $\beta$ (Derynk et al. 1985), Müllerian inhibiting substance (Cate et al. 1986), inhibin (Mason et al. 1985), and bone 
morphogenesis proteins (Wozney et al. 1988). The polypeptide encoded by the Vgl RNA, which is localized to the vegetal pole of Xenopus oocytes, is also a member of this family (Weeks and Melton 1987). These factors, participating in a wide range of developmental processes, are believed to act extracellularly through binding to receptors on target cells. Because the structure of the $d p p$ polypeptide contains an amino-terminal signal sequence, amino-glycosylation sites, and no transmembrane domains, we have proposed that the $d p p$ product, by analogy with the vertebrate members of the TGF- $\beta$ family, acts as a secreted factor during Drosophila development (Padgett et al. 1987). The strongly ventralized phenotype displayed by $d p p^{\text {Hin }}$ homozygotes and the early requirement for $d p p$ activity suggest further that intercellular communication plays an early and important role in dorsal-ventral determination.

As with several other pattern-determining genes in Drosophila (Baker 1988; Carroll and White 1989), dpp is reactivated in a position-specific fashion during later stages of development (L.M. Posakony et al., in prep.). Its expression is essential for the growth and differentiation of the 19 primordia called imaginal discs, which differentiate into the epidermis of much of the adult, including all appendages. Analysis of $d p p$ mutations defective in disk development suggests that $d p p$ is involved in the elaboration or interpretation of proximal-distal positional information within the developing appendages. Clonal analysis has revealed that $d p p$ is not cell autonomous in the imaginal discs (L.A. Posakony et al., in prep.). Finally, $d p p$ expression is also required for normal larval development, although little is known of its involvement in this process.

The diversity of developmental requirements of $d p p$ in different tissues and at different times is mirrored in the complex genetic organization of the locus. Three major regions of the gene have been defined by genetic criteria [shortvein (shv); haploinsufficiency (Hin); imaginal disk specific-disk]. From the complementation properties of partial deletions of $d p p$, these three regions map in the order shv, Hin, and disk (Spencer et al. 1982; Segal and Gelbart 1985). In this paper, we molecularly map the lesions of 60 mutations within the gene and present a detailed transcriptional analysis of $d p p$. From our observations, we infer that $d p p$ is likely to encode a single polypeptide product. We also conclude that the bulk of the $>55-\mathrm{kb}$ gene consists of arrays of cis-regulatory sequences. A striking organizational feature of $d p p$ is that the disk region consists of cis-regulatory information spread over $\geqslant 25 \mathrm{~kb}$ downstream $\left(3^{\prime}\right)$ of the $d p p$ transcription unit.

\section{Results \\ The cloning of $\mathrm{dpp}$}

Single-copy sequences from a clone recovered by transposon tagging were used to initiate a chromosome walk, which eventually encompassed $140 \mathrm{~kb}$ (for details, see Experimental procedures). All cytologically rear- ranged $d p p$ mutations that were analyzed have a molecular breakpoint within this walk. At one step in the chromosome walk, a cross-hybridizing phage from a different genomic region was obtained. Cross-hybridization is due to the presence of tRNA ${ }^{\mathrm{Tyr}}$ genes within these clones. Further analysis has demonstrated that two tRNA $^{\text {Tyr }}$ genes reside within $d p p$ (B. Suter et al., in prep.).

\section{Organization of the dpp gene}

Mutant lesions were localized on the molecular map by a combination of chromosomal in situ hybridization, whole genome Southern blotting, and restriction mapping of cloned mutant DNA (see Experimental procedures). From an examination of $69 d p p$ alleles (Table 1), 60 possessed molecular lesions within a $38.5-\mathrm{kb}$ interval of the 22F1,2 chromosome walk (Fig. 1). In many cases, the lesion involves a single breakpoint in the $d p p$ gene, reattaching it to some other region of the genome; such lesions will be referred to as breakpoint alleles. Strikingly, mutations producing similar phenotypes cluster on the molecular map. Thus, the genetically characterized functions of $d p p$ correspond to discrete molecular regions of the gene. These subdivisions are named for the phenotypes of mutations falling in these regions. The molecular and phenotypic properties of these subdivisions are described in the next section. For simplicity, $d p p$ mutations will be referred to by only their superscript designations (e.g., $d 21$ refers to $d p p^{d 21}$ ).

\section{shv region}

The shv region is named after the phenotype engendered by all $s h v$ mutations, in which the longitudinal veins of the wing are reduced or absent (Segal and Gelbart 1985). Twenty-three $s h v$ mutations have been analyzed, 19 of which are associated with molecular breakpoints in $d p p$ (Segal and Gelbart 1985). These shv alleles can be subdivided into several categories on the basis of their other phenotypic properties. The 19 breakpoint alleles fall into three classes. The two weakest alleles, s11 and s22, survive at least until pharate adult (i.e., late pupation) in heterozygous combinations with all other $s h v$ mutations (Segal and Gelbart 1985; D. Hursh and R. Ray, pers. comm.); because of their survival to pharate adult, they are termed $s h v-p$ mutations. Surviving adults exhibit strong wing venation defects and also show a reduction of the maxillary palps and vibrissae of the head capsule. The other 17 breakpoint mutations cause early larval lethality; these are termed $s h v-1$ alleles, indicating their larval lethality. Some of these alleles fully complement all disk mutations (shv-lc alleles), whereas others (shvInc) do not. Mutations in this latter class exhibit mild shv and disk phenotypes in trans-heterozygotes with severe disk mutations. One of the $s h v \cdot \ln c$ alleles, $s 19$, is exceptional in that it completely fails to complement all disk region mutations. One possible explanation for the severity of the $s 19$ interaction with disk alleles is that it contains a second lesion in the disk interval; however, 
Table 1. Molecular properties of the 13 categories of dpp mutations analyzed in this study

\begin{tabular}{|c|c|c|c|c|}
\hline \multirow[b]{2}{*}{$\begin{array}{l}\text { Mutant } \\
\text { class }\end{array}$} & \multicolumn{3}{|c|}{ Designations of alleles that are molecularly } & \multirow[b]{2}{*}{$\begin{array}{l}\text { Total number } \\
\text { of alleles }\end{array}$} \\
\hline & normala & $\begin{array}{l}\text { small deletions } \\
\text { or insertions } \mathbf{s}^{\mathrm{a}, \mathrm{b}}\end{array}$ & rearranged ${ }^{c}$ & \\
\hline shv-w & - & s1 s6 & - & 2 \\
\hline shv-p & - & - & s11 s22 & 2 \\
\hline shv-lc & - & - & $s 2 s 3^{\mathrm{d}} s 7 s 12 s 15 s 20 s 23^{\mathrm{e}} s 26 s 28^{\mathrm{d}}$ & 9 \\
\hline shv-lnc & s8 s10 & - & s4 s5 s13 s14d s18 s19 s21 s25 & 10 \\
\hline Hin & Hin 48 Hin 88 & Hin46 Hin47 Hin61 & $\operatorname{Hin} 37^{\mathrm{e}} \operatorname{Hin} 45$ & 7 \\
\hline hin-r & $\begin{array}{l}\text { hin-r4 hin-r27 hin-r56 } \\
\text { hin-r89 hin-r } 90\end{array}$ & - & - & 5 \\
\hline emb & - & $e 87$ & - & 1 \\
\hline $\mathrm{t}$ & - & - & $t 24 t 63$ & 2 \\
\hline disk-V & - & - & $d 12 d 13 d 21 d 26$ d29 d35 d52 d70 d72d & 9 \\
\hline disk-III & - & - & 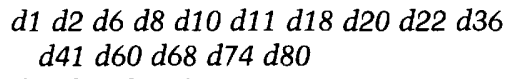 & 15 \\
\hline disk-II & - & - & $d 5^{\mathrm{e}} d 28 d 50 d 66$ & 4 \\
\hline disk-ho & - & d-ho d-hb1-blk d-hb3-blk & - & 3 \\
\hline disk-blk & - & $d-b l k$ & - & 1 \\
\hline
\end{tabular}

All of the alleles in this category are cytologically normal.

'This category includes deletions of small portions of the $d p p$ gene and/or mobile element insertions.

cUnless otherwise noted, all alleles in this category have cytologically detectable rearrangements. Unless otherwise noted, sequences juxtaposed to $d p p$ are euchromatic.

'In this breakpoint allele, $d p p$ sequences were juxtaposed to centric heterochromatin.

'This allele is cytologically normal.

no alterations in the restriction maps of the Hin or disk regions have been observed.

All 19 of the $s h v$ breakpoint alleles have their restriction map alterations in the interval between 72.5 and 83.0 on the molecular map (Fig. 1). The three classes of breakpoint alleles map in discrete parts of this region: the two $s h v$-p alleles, $s 11$ and $s 22$, break between 72.5 and 73.2, the larval lethal shv-lc alleles fall between 73.2 and 80.1 , and the $s h v-\ln c$ alleles fall between 79.3 and 83.0. The $s h v-\ln c$ allele $s 5$ falls just distal to the two most proximal $s h v-l c$ alleles, $s 15$ and $s 23$. With this one exception, the three phenotypic classes of breakpoint alleles fall into nonoverlapping regions of the molecular map. The most severe alleles $(s h v-\ln c)$ lie closest to the Hin region, whereas the weakest alleles, shv-p, fall most distally.

The remaining four alleles are cytologically normal. Two, $s 8$ and $s 10$, behave as shv-lnc mutations; no changes in their restriction maps in the 67-83 region were detected. $s 1$ and $s 6$ define a fourth category of $s h v$ mutation, which we designate the $s h v-w$ class. Both alleles are fully viable and only affect wing venation of the adult. $s 1$ and $s 6$ are associated with small deletions of 0.9 and $4.5 \mathrm{~kb}$, respectively. These deletions remove DNA sequences from the distal end of the shv region.

\section{Hin region}

Seven Hin mutations have been identified, and all behave as if they are null for every $d p p$ function (Irish and Gelbart 1987; Posakony 1987). Embryos that are homozygous for Hin alleles fail to complete germ-band exten- sion and eventually die with a strongly ventralized phenotype in which no derivatives of the dorsal epidermis are formed (Irish and Gelbart 1987). These mutations also exhibit a haploinsufficiency (from which the name Hin derives/, in which $\mathrm{Hin} /+$ embryos die with a weakly ventralized phenotype. Occasional escapers from the haploinsufficiency permit the examination of $\mathrm{Hin} /$ $s h v$ and Hin/disk genotypes; such animals exhibit strong $s h v$ - and disk-related phenotypes, respectively.

Of the seven Hin mutations, two (Hin48 and Hin88) have normal restriction maps in the entire molecular interval that was examined (76-93). The other five have discernible molecular lesions that fall within the 83.5-89.0 interval. Hin 37 and Hin 45 are associated with rearrangement breakpoints. Hin 46 , Hin 47 , and Hin61 are small deletions of $0.3,0.6$, and $2.0 \mathrm{~kb}$, respectively.

Five ethyl methanesulfonate (EMS)-induced recessive embryonic lethal alleles of $d p p$ have been proposed to be leaky (hypomorphic) mutations in the Hin region (Irish and Gelbart 1987) (hin-r4, hin-r27, hin-r56, hin-r89, and hin-r90). These mutations have normal restriction maps in the 76-93 region.

\section{A mutation specific for embryogenesis}

One mutation, $e 87$ (Irish and Gelbart 1987), in hin-r/e87 heterozygotes, has a recessive embryonic lethal phenotype indistinguishable from that of hin-r homozygotes; however, $e 87$, unlike hin-r mutations, completely complements lesions in the $s h v$ and disk regions. Furthermore, e87 homozygotes are viable and fertile. Thus, it appears to be a leaky embryonic stage-specific mutation. 
dpp gene structure

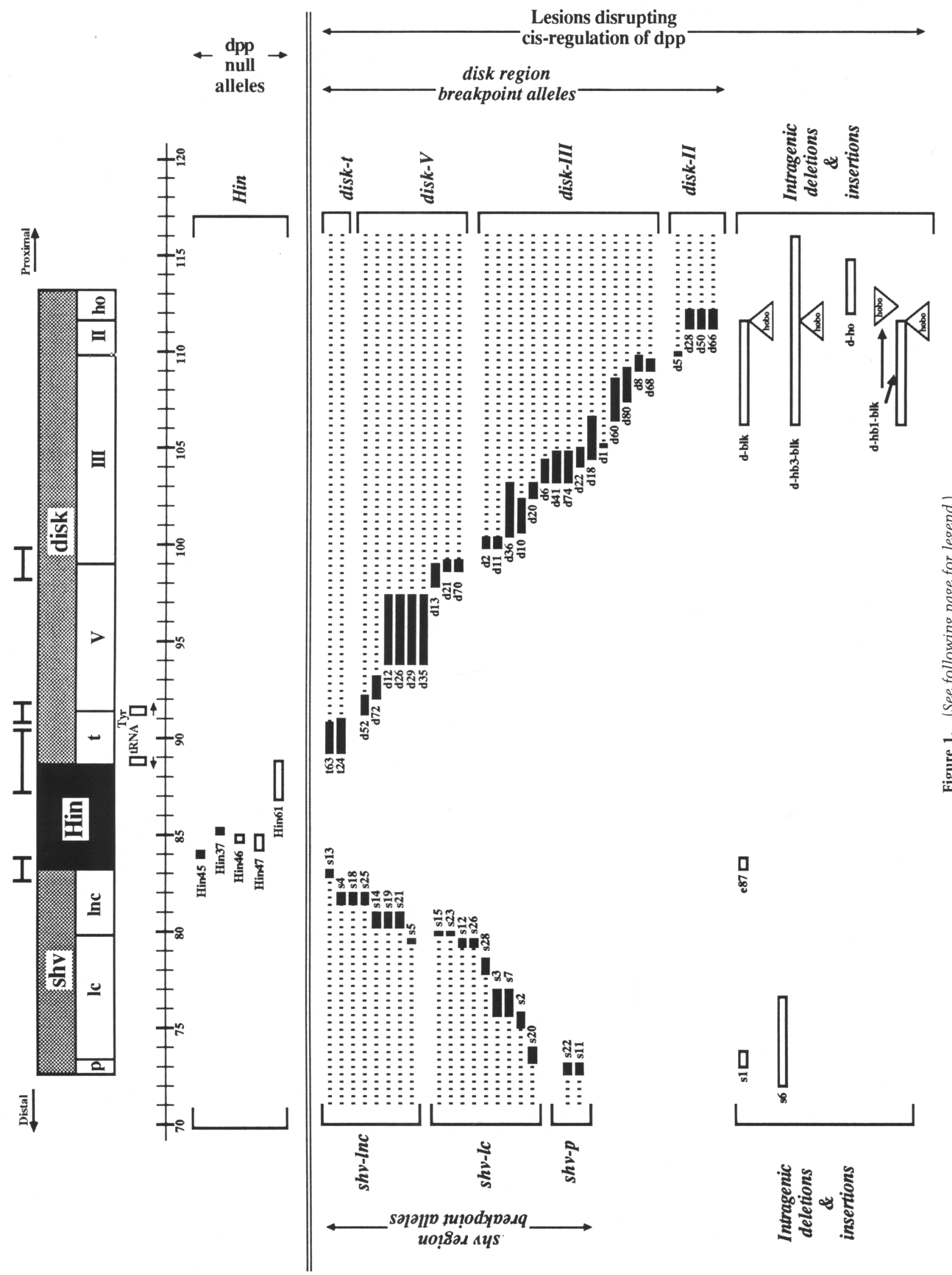


St. Johnston et al.

This mutation has been cloned and is associated with a deletion of $\sim 0.5 \mathrm{~kb}$ between 83.2 and 83.7 , in the interval bordering the shv and Hin regions.

\section{$t R N A^{T y t}$ region}

The two tRNA ${ }^{\mathrm{Tyr}}$ genes within $d p p$ reside at 88.8 and 91.6 (B. Suter et al., in prep.), falling between the Hin and disk regions. Two mutations, $t 24$ and $t 63$, have breakpoints in the interval between these two tRNA ${ }^{\text {Tyr }}$ genes. The trans-heterozygote $t 24 / t 63$ dies during the larval period. This larval lethality distinguishes these two alleles from those breakpoint mutations in the neighboring Hin and disk-V regions. Mutations in the disk-V region (see below) survive at least to pupariation in all trans-heterozygous combinations, whereas all Hin region breakpoints exhibit a dominant embryonic lethal phenotype. These differences suggest that $t 24$ and $t 63$ may define a distinct category of $d p p$ mutation, however, it is unclear whether this category reflects perturbations in the expression of the tRNA genes (see Discussion).

\section{disk region}

Most disk region mutations elicit multiple defects in the adult epidermal derivatives of the imaginal discs (Spencer et al. 1982). Phenotypic abnormalities elicited by mutations in this region can also be observed in the imaginal discs of the late third-instar larva (Spencer et al. 1982; Bryant 1988).

The disk region lesions associated with breakpoints have been grouped into three major classes (Spencer et al. 1982 and unpubl.). The most severe are the disk-V alleles. Larvae homozygous for such mutations possess rudimentary imaginal discs; these animals subsequently die during pupariation (K. Madhavan, pers. comm.). The intermediate disk-III alleles produce adults lacking distal portions of the appendages derived from $\geqslant 15$ of the 19 imaginal disks. The mildest class comprises the disk-II mutations. disk-II homozygotes lack large portions of the wing blade, the halter, and the male genitalia. The phenotypes elicited by mutations in these three classes appear to form a nested array in that mutations in the more severe class not only delete all of the structures removed by a milder mutation but also delete more pattern elements derived from a given disk and affect more of the disks.

Mutations of the disk-V, disk-III, and disk-II classes are associated either with inversions or translocation breakpoints within the disk region or with deletions beginning within the disk region and extending proximally through one or more adjacent vital genes. The disk- $V$ alleles break closest to the Hin region, within the 92-99 interval, whereas disk-III alleles fall within 100.0110.1 , and disk-II alleles fall within 110.2-112 (Fig. 1). Thus, the disk-V, disk-III and disk-II regions do not overlap. The sizes of the disk-V, disk-III, and disk-II regions $\{\sim 7,10$, and $2 \mathrm{~kb}$, respectively) parallel the number of induced mutations of each type that have been recovered in screens capable of identifying all three classes of alleles $(19,26$, and 5, respectively) (Spencer et al. 1982; Irish and Gelbart 1987; D. Segal and W. Gelbart, unpubl.).

In addition to the mutations associated with breakpoints, four cytologically normal disk alleles are associated with small deletions or transposon insertions (Fig. 1). Without exception, these mutations produce milder phenotypes than rearrangement mutations which break at similar molecular positions. These weaker alleles include $d$-blk, that causes a reduction in eye size, and three alleles that cause the wings to be held out horizontally from the body, $d$-ho, $d$-hb1-blk, and $d$-hb3-blk (Blackman et al. 1987). The two mutations $d$-hb1-blk and $d$-hb3-blk arose on a $d$-blk parental chromosome and therefore also engender the $d$-blk eye phenotype. The molecular defects in these four disk-specific mutations have been described elsewhere (Blackman et al. 1987) and are diagrammed in Figure 1.

\section{Transcriptional analysis of the dpp gene}

To analyze the transcription of $d p p$, DNA spanning all of the mutant breakpoints (from 68 to 119 on the molecular map/ was divided into fragments of $\geqslant 2 \mathrm{~kb}$, subcloned in both orientations into M13 or SP6 vectors and used to probe Northern blots of staged Drosophila RNAs. The probes from the shv and Hin regions hybridized to several transcripts, all of which are transcribed from distal to proximal (from $s h v$ toward disk).

The Hin region probes hybridized to three major transcripts, A, B, and C, which are $3.3,3.8$, and $4.3 \mathrm{~kb}$ long,

Figure 1. A molecular map of the $d p p$ gene. On the basis of the phenotypes of mutations falling in particular regions, $d p p$ can be subdivided into several functional domains. The domains are depicted at the top. Uncertainties in the locations of the boundaries separating the domains are indicated by the horizontal bars above the domain map. The distal and proximal arrows orient the walk on the standard chromosome map; they point in the directions of the telomere of chromosome arm $2 \mathrm{~L}$ and the centromere of chromosome 2, respectively. The scale 70-120 represents nucleotide positions along the chromosome walk (in kb). The locations of the two tRNA $^{\text {Tyr }}$ genes are indicated above the scale. The locations of $60 \mathrm{dpp}$ mutations are positioned along the chromosome walk through $22 \mathrm{Fl}$,2. Mutational positions are represented according to type of mutant lesion. The locations of $d p p$ translocation or inversion breakpoints separating the $d p p$ gene into two nonadjacent fragments are depicted as solid bars. The uncertainty in the location of each breakpoint is represented by the length of the solid bar. The dotted line adjacent to each solid bar represents the $d p p$ functions inactivated by the breakpoint. Mutations associated with deletions of material within the 70-120 region of the walk are depicted as open bars. The lengths of the open bars indicate the sizes of the deletions. The triangles represent insertions of a 1.9-kb hobo element DNA within $d p p$. $d$-blk and its two derivatives, $d$-hb1-blk and $d$-hb3-blk, are intragenic deletions associated with hobo elements located at the sites of the deletion endpoints; this element was present at 111.4 in their $d p p^{+}$parental strain (Oregon- $R^{\text {Sparrow }}$ strain) (Blackman et al. 1987). 


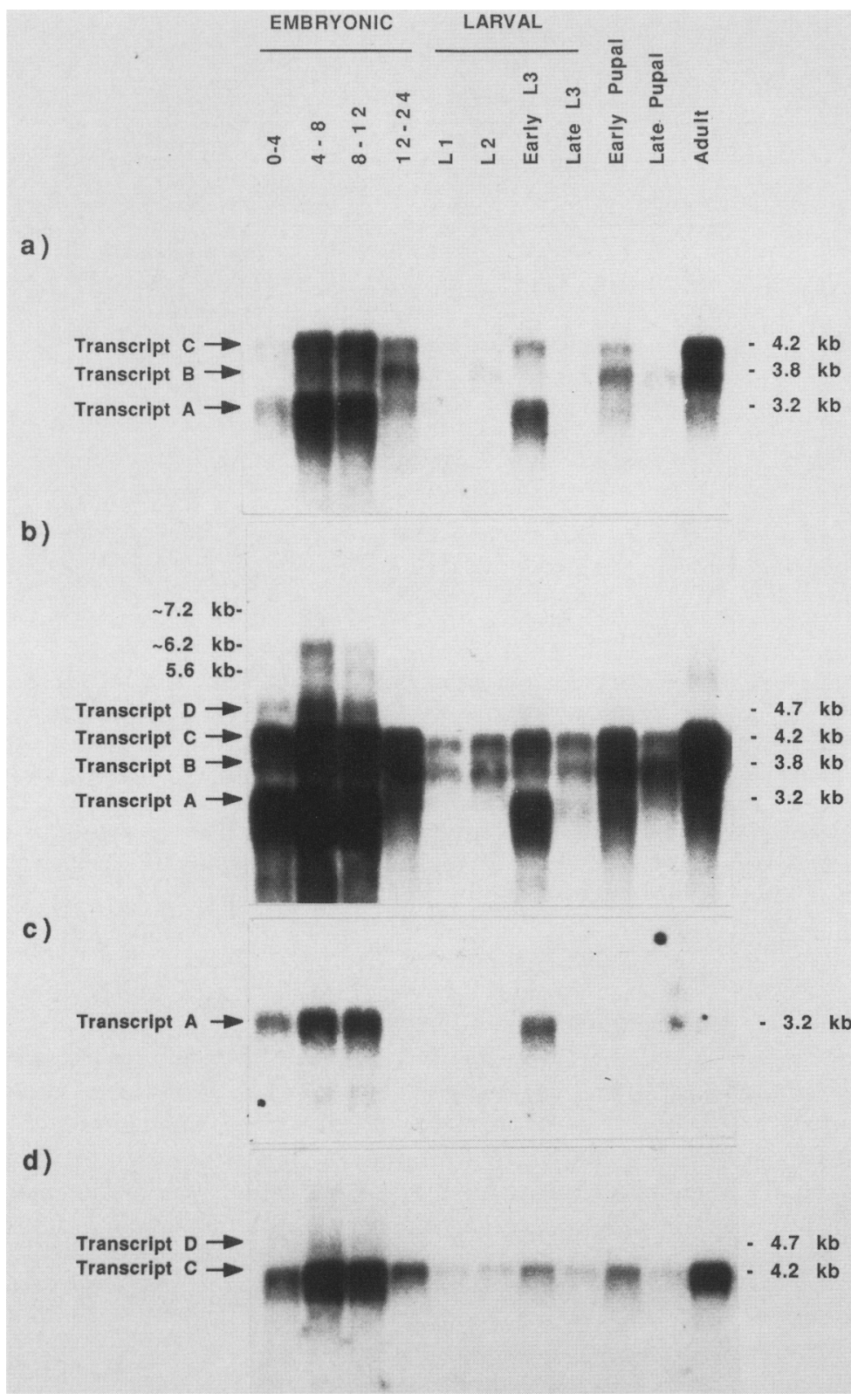

Figure 2. Transcripts from $d p p$. Developmental Northern blots were hybridized with single-stranded DNA probes, as described in Experimental procedures. All of the probes shown hybridize to RNAs transcribed from distal to proximal. RNA was prepared from staged animals reared at $25^{\circ} \mathrm{C}$. The embryonic lanes contain RNA isolated from 0- to 4-, 4- to 8-, 8- to 12-, and 12- to 24-hr-old embryos, respectively. L1, L2, and L3 refer to the first-, second-, and third-larval instars. (a) 83.6-84.7 Hin region probe; $(b)$ 83.6-84.7 Hin region probe; $(c)$ 83.5-83.8 probe, specific to transcript $\mathrm{A}_{;}(d) 80.2-81.3$ shv region probe. respectively, as well as to some minor species (Fig. 2a). Transcript A is the most abundant RNA in early embryos. It is present in 0- to 4-hr embryos, reaches a peak between 4 and $12 \mathrm{hr}$ of development, and disappears after this time; it is re-expressed in early third-instar larvae. Transcript B is first detectable in $8-$ to $12-\mathrm{hr}$ embryos and remains present throughout the rest of development, with highest levels in 12- to 24-hr embryos and early pupae. Transcript $C$ has an early accumulation profile similar to transcript $A$ but remains on throughout development and is present at high levels in adult flies. In longer exposures, several larger species can also be detected, including a minor 4.6-kb RNA, transcript $D$, which is expressed most strongly in early embryos (Fig. 2b). The other less abundant RNAs range in size between 5.6 and $7.2 \mathrm{~kb}$ and may be unspliced precursors of the smaller RNAs.

To investigate the structures of these transcripts, several cDNA libraries, made from RNAs of different developmental stages, were screened. More than 30 independent cDNA clones were recovered. Their structures (Fig. 3 ) indicate that each of the $d p p$ RNAs possesses two common $3^{\prime}$ exons in the Hin region but has one of at least five alternative $5^{\prime}$ exons. Sixteen cDNA clones (those whose names begin with $B$ in Fig. 3) were recovered from libraries that were constructed by using a directional cloning strategy ensuring that the $3^{\prime}$ end of the RNA is represented in the cDNA (Brown and Kafatos 1988). All of these clones are derived from RNAs that terminate at one of three alternative poly|A addition 


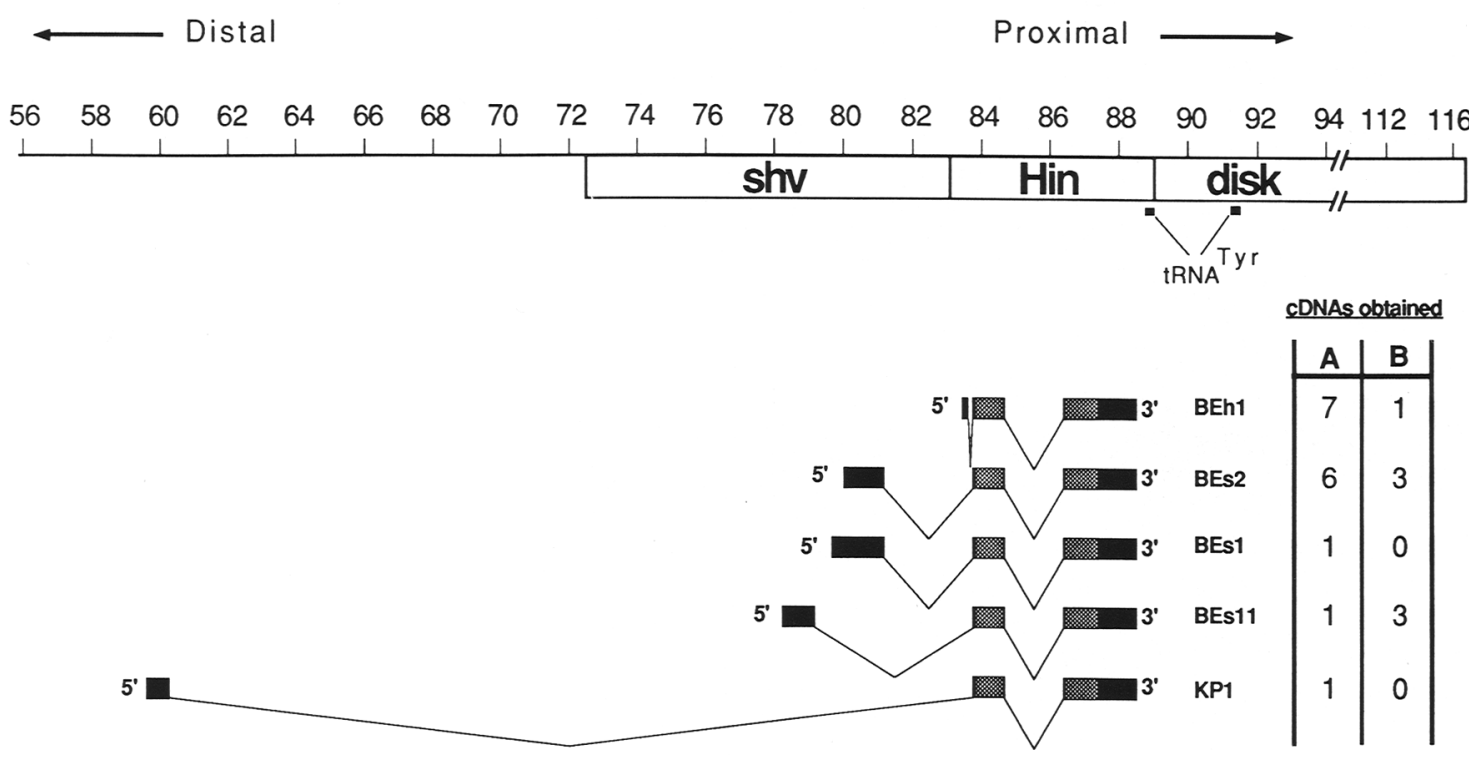

Figure 3. The structures of cDNA clones from $d p p$. The exonic structure of the longest member of each class of cDNA is diagrammed beneath a genomic map of the distal portion of $d p p$. The stippled region within each cDNA corresponds to the position of the protein-coding region, on the basis of sequence of two partial cDNAs in the BEs2 class (Padgett et al. 1987). Column A shows the total number of cDNAs that are as complete as the longest member of each class; column $B$ lists the number of partial cDNAs in each class. In addition to these cDNAs, several clones have been isolated that only contain sequences from the two common exons in the Hin region. Because these clones do not contain $5^{\prime}$ exon material, they cannot be assigned to one of these classes. The BE cDNAs were all recovered from a 4- to 8-hr embryonic library (Brown and Kafatos 1988). KP1 was isolated from a pupal cDNA library (Poole et al. 1985). Transcripts A-D relate four of these cDNA classes to RNAs defined by the Northern blots in Figs. 2 and 4.

sites that lie between 88.4 and 88.7 (data not shown). The most proximal poly(A) addition site lies 100 bases distal to the $3^{\prime}$ end of the tRNA ${ }^{\text {Tyr }}$ gene at 88.5 that is transcribed in the opposite direction.

Seven cDNA clones, including BEh1, contain a small $5^{\prime}$ exon at $\sim 83.5$. When DNA from this exon is used to probe a Northern blot, only transcript $A$ is detected (Fig. $2 c)$. Because several independent cDNAs have been recovered with similar $5^{\prime}$ extents and more distal probes do not hybridize to this message, it seems probable that these cDNAs are essentially full-length copies of the 3.3-kb RNA and that this transcript is entirely encoded within the Hin region. This has been confirmed in S1 protection experiments (St. Johnston 1988; data not shown).

CDNA BEs2 and six other cDNA clones all contain a $1.1-\mathrm{kb}$ exon that extends from 80.1 to 81.2 . The 80.2-81.3 genomic HindIII fragment hybridizes strongly to transcript C (Fig. 2d), whereas the adjacent 79.5-80.2 fragment recognizes this RNA much more weakly (Fig. 4a). More distal probes do not hybridize to this transcript. These results suggest that BEs2 extends close to the $5^{\prime}$ end of transcript $C$, and this has been confirmed in S1 protection experiments (St. Johnston 1988). The same two genomic fragments also hybridize to the much rarer RNA, transcript $\mathrm{D}$; however, the more distal probe (79.2-80.2) recognizes this RNA more strongly relative to its abundance than it does transcript $C$, suggesting that $\mathrm{D}$ extends further distally. This conclusion is supported by the observations that cDNA BEs1 contains a further $200 \mathrm{bp}$ of sequence $5^{\prime}$ to the end of transcript $\mathrm{C}$, and that this $200 \mathrm{bp}$ hybridizes specifically to transcript $\mathrm{D}$ (data not shown).

The 79.5-80.2 probe also hybridizes to two fainter bands that are only visible in the lanes containing early embryonic RNA. More distal probes, such as $77-78$, also hybridize to a number of transcripts that are only expressed in early embryos (Fig. 4b). Because the exposures required to detect these transcripts are an order of magnitude longer than those for the major RNA species, these RNAs are of very low abundance. Three cDNAs, the longest of which is BEs 11 , contain $5^{\prime}$ exons in this region.

cDNA KP1, which was isolated from an early pupal cDNA library (5.5-7.5 days post-egg lay; Poole et al. 1985), contains a 5 ' exon that lies at coordinate 60 of the $d p p$ molecular map. A single-stranded probe from this region hybridizes specifically to transcript $B$, which therefore contains a $5^{\prime}$ exon that is separated from the common Hin region exons by an intron of $23 \mathrm{~kb}$ (Fig. 4c). Distal shv probes, such as the one used in Figure $4 \mathrm{~b}$, detect a high-molecular-weight RNA that is expressed in 12- to 24-hr embryos, pupae, and adults, the same temporal profile as transcript $B$. This high-molecular-weight RNA may represent unspliced or partially spliced precursors of transcript $B$.

DNA spanning the entire disk region from 93 to 119 on the molecular map has also been used to probe developmentally staged Northern blots. Almost all fragments displayed no hybridization signal. One fragment, extending from 109.5 to 111.0 , hybridized to three transcripts (data not shown). These RNAs hybridize to the 
a)

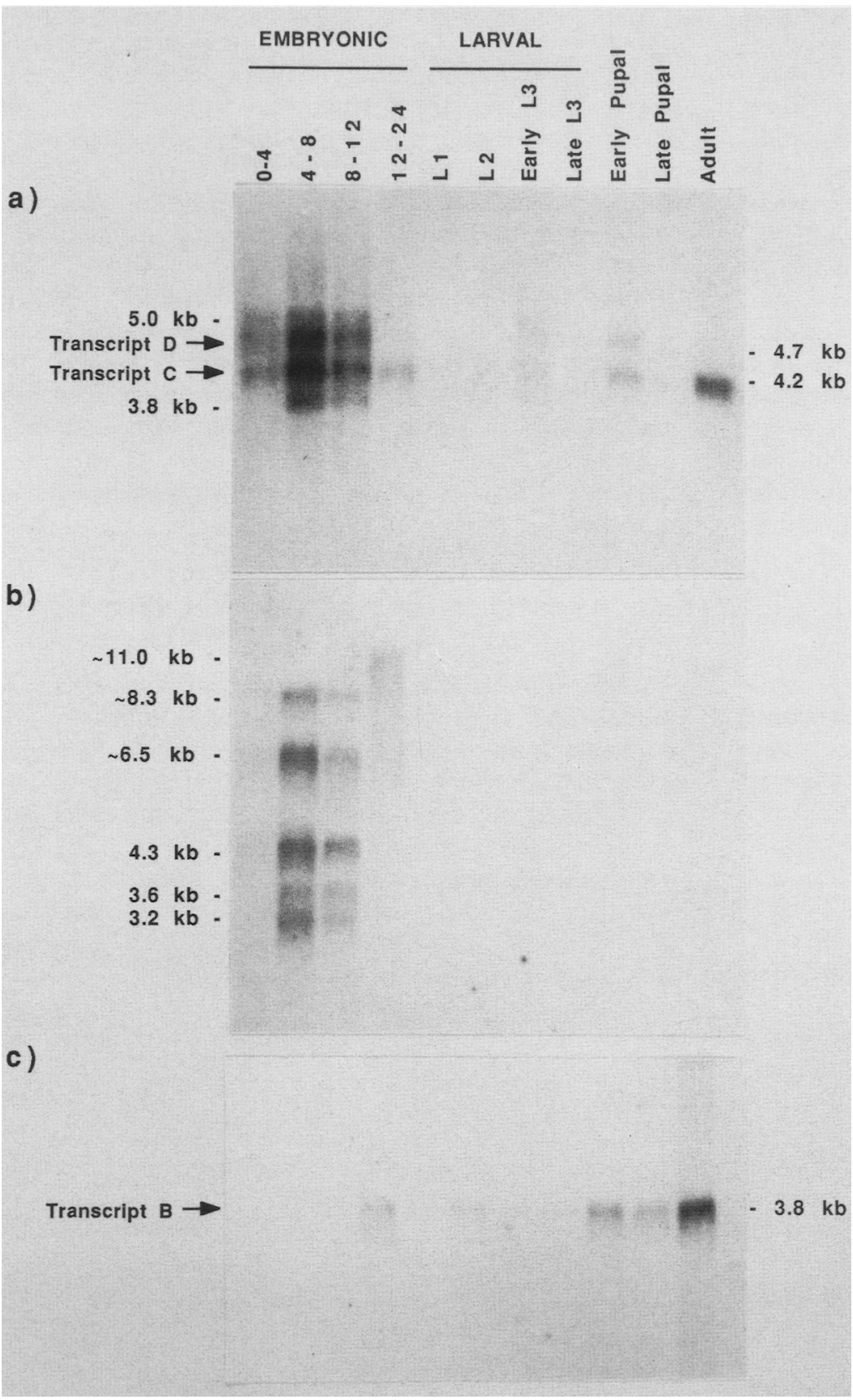

Figure 4. Northern blots hybridized with distal shv region probes. All experiments were performed as described in Experimental procedures and in Fig. 2. (a) 79.3-80.2 probe; (b) 77.1-78.5 probe; (c) $59.1-60.7$ probe. opposite strand relative to the shv-Hin RNAs. For two of these transcripts $(1.1$ and $3.8 \mathrm{~kb})$, it is possible that their signal is due to cross-hybridization with RNAs that are transcribed elsewhere in the genome, as very long exposures are required to see these bands. The third transcript $(0.8 \mathrm{~kb})$ also hybridized to the adjacent $111.0-$ 113.2 region. It showed no temporal variation in expression. Several cDNA libraries have been screened with DNA from the disk region, but no clones have been detected.

\section{Discussion}

The molecular characterization of $d p p$ clarifies our view of this locus and provides us with a striking and, in some respects, novel general picture of the organization and regulation of the gene and its product. In developing a model of the gene, several features have to be taken into account. One major feature of $d p p$ is that mutations with similar phenotypes cluster and define eight distinct regions on the molecular map (Fig. 1). These regions are basically continuous; it does not appear as if there are any large regions within $d p p$ that are mutationally silent. Many of the $d p p$ mutations are associated with gross chromosomal rearrangements, in that exogenous DNA is juxtaposed to normal $d p p$ sequences. The clustering of breakpoints according to phenotype strongly suggests that it is the position of the breakpoint within $d p p$ that determines the type and severity of phenotypic disruption caused by a particular mutation and, hence, 
that the eight distinct regions must reflect important features of the molecular organization of $d p p$.

The transcriptional analysis of $d p p$ has revealed the existence of a number of different RNAs encoded by the shv and Hin regions of the gene. Although the structure of some of the minor transcripts still remains obscure, it is clear that all of the major RNA species share two common exons that reside within the Hin region but differ in their $5^{\prime}$ exons. The $5^{\prime}$ exon of transcript $\mathrm{B}$ lies at coordinate 60 on the molecular map, $12 \mathrm{~kb}$ distal to the most distal $s h v$ mutant lesion. This gives a minimum size of the $d p p$ gene of $51.7 \mathrm{~kb}(60.0-111.7)$, although the 5 ' end of transcript $B$ has yet to be mapped and could extend even further distally.

The phenotypes of Hin, shv, and disk alleles indicate that $d p p$ expression is required for dorsal determination in the embryo, development through the larval period, and morphogenesis of the imaginal discs. This variety of phenotypes suggests that $d p p$ transcripts must be expressed at several stages of development in a temporally and spatially complex pattern. Tissue in situ hybridizations to embryos (St. Johnston and Gelbart 1987) and larvae (L.M. Posakony, L.A. Raftery, R.D. St. Johnston, and W.M. Gelbart, in prep.) have shown that this is the case and reveal further components of the $d p p$ expression pattern that would not have been predicted from the mutant phenotypes. Although it is not yet known which portions of the total $d p p$ expression pattern are contributed by each transcript, it is clear these RNAs are regulated differently, as transcripts $A, B, C$, and D all have distinct developmental profiles on Northern blots (see Figs. 2 and 4). Because the structure of these RNAs indicates that each has a separate promoter, this regulation probably occurs at the level of transcript initiation, with each promoter being controlled by a different combination of cis-regulatory elements.

The sequencing of cDNAs derived from transcript $\mathrm{C}$ reveals that the two exons in the Hin region contain a long open reading frame (ORF), whereas the 5' exon of this RNA is entirely noncoding (Padgett et al. 1987). Because each of the major $d p p$ transcripts contains these two Hin region exons, all of these RNAs must encode similar proteins. It is possible that the alternative $5^{\prime}$ exons contain protein-coding information, and that these other transcripts produce polypeptides with different amino termini. However, the homology to TGF- $\beta$ lies at the carboxy-terminal end of the ORF. Thus, the structures of the $d p p$ transcription units and the sequences of the cDNAs suggest that all $d p p$ RNAs are likely to encode the same secreted factor and that the pleiotropic effects of $d p p$ mutations reflect the multiple functions of this factor during development. Therefore, $d p p$ clearly can be categorized as a complex gene, as opposed to our previous view that it was likely to be a gene complex (i.e., a cluster of related transcription units; Gelbart et al. 1985).

Hin mutations disrupt the protein-coding information of dpp

Hin alleles, which are haplolethal, and hin- $r$ alleles, that are haploviable, behave as if they are null and hypomorphic, respectively, for all $d p p$ functions (Irish and Gelbart 1987). The Hin mutations with detectable molecular lesions alter information common to all of the $d p p$ transcripts. Hin46, Hin47, and Hin61 are small deletions, each removing part of the ORF, whereas Hin37 and Hin 45 are rearrangements that interrupt all of the $d p p$ transcription units. Thus, the amorphic nature of these mutations can be explained by the fact that they disrupt all $d p p$ transcripts and/or polypeptides.

We failed to identify any restriction map alterations in the Hin region of Hin 48, Hin 88, and five hin- $r$ alleles. To explain their global effects, we think it most likely that they are point or pseudopoint mutations within the protein-coding sequences of the gene. The leaky nature of the hin-r alleles further suggests that they are missense mutations.

\section{Hin region also contains cis-regulatory sequences necessary to fulfill the role of dpp in embryogenesis}

The only molecularly visible mutations that exhibit embryonic phenotypes fall in the Hin region. Furthermore, all breakpoints in the shv region are viable in homozygotes through the embryonic period and are haplosufficient. Thus, by breakpoint analysis, the $d p p$ functions required in homozygotes for development through embryogenesis map proximal to $s 13(82.7)$ and distal to $d 52$ and $d 72(92.0)$. By the same reasoning, the dominant haploinsufficiency of $d p p$ falls between $s 13$ (82.7) and $t 63$ (90.6). Consistent with this information, all of the embryonic abnormalities elicited by Hin and hin- $r$ alleles are rescued by germ line transformants containing the 82.6-90.8 region (Hoffmann and Goodman 1987).

These results indicate that the Hin region contains sufficient information to fulfill all of the embryonic $d p p$ functions that are necessary for survival to the larval period. This region includes the entire transcription unit of transcript $A$ and the more distal of the two tRNA ${ }^{\text {Tyr }}$ genes (Y22Fb, B. Suter et al., in prep.). Although it is formally possible that the tRNA ${ }^{\mathrm{Tyr}}$ gene provides these embryonic $d p p$ functions, we consider this alternative highly improbable because all of the molecularly characterized Hin alleles fall within transcript A, up to $5 \mathrm{~kb}$ downstream of the $3^{\prime}$ end of the Y22Fb tRNA ${ }^{\text {Tyr }}$ gene. Also, there are at least seven other tRNA ${ }^{\text {Tyr }}$ genes in the genome, which could supply the necessary tRNA during this stage of development. It should be noted that the $d p p$ polypeptide is not unusually rich in tyrosine residues (12 tyrosines in a sequence of 588 amino acids; Padgett et al. 1987).

Most probably, the embryonic functions of $d p p$ are provided by transcript $\mathrm{A}$, the only intact $d p p$ transcript encoded by the 82.6-90.8 transposon or in many embryonic viable $s h v$ breakpoint alleles, such as $s 13$. Consistent with this view, transcript $A$ is the most abundant of the early embryonic transcripts. The 82.6-90.8 transposon must also contain the appropriate cis-regulatory elements controlling those aspects of the expression of transcript $\mathrm{A}$ that are needed for embryonic survival. For 
example, some of these elements must be able to direct the early dorsal expression of $d p p$ that is required for normal germ-band elongation (St. Johnston and Gelbart 1987). Because $d p p$ is one of the first zygotic genes to be activated, this early expression may be regulated directly by the maternal dorsal-ventral signals. It has been proposed that this maternal information is provided by a gradient of the dorsal protein in blastoderm nuclei (Roth et al. 1989; Rushlow et al. 1989; Steward 1989|.

\section{Relationship of the surrounding shv and disk regions to Hin}

Mutations in the shv and disk region contrast with those in the Hin region in several respects. (1) All shv and disk mutations affect specific subsets of the phenotypes controlled by the $d p p$ gene; Hin and hin-r mutations affect all $d p p$ phenotypes. (2) Virtually all shv and disk mutations are gross alterations detectable by whole genome Southern blot analysis. Many lesions in the Hin region have normal restriction maps. (3) There is a correlation between the severity of a shv or disk breakpoint mutation and its distance from the Hin region. The farther the $s h v$ or disk mutation is from Hin, the milder its phenotype. (4) Breakpoint alleles within the shv and disk regions are more severe than small deletions removing the corresponding regions; breakpoint and deletion mutations of the Hin region are equivalent in severity.

The structures of the $d p p$ transcription units suggest that most or all of the protein-coding capacity of the gene lies within the Hin region exons. The extremely low frequency at which point mutations can be recovered in the shv and disk regions (from among $>100 \mathrm{sh} v$ and disk mutations that have been generated, we have recovered no more than 2 , and possibly no such point mutations) also indicates that these parts of $d p p$ do not contain any extensive or essential protein-coding regions. Thus, the $s h v$ and disk alleles appear to affect $d p p$ functions without altering the structure of the $d p p$ protein product. To rationalize the genetic and phenotypic properties of mutations in the shv and disk regions, we propose that these alleles affect the regulation of the complex spatial and temporal pattern of $d p p$ expression. The $s h v$ and disk alleles can affect $d p p$ expression either by disrupting a subset of the $d p p$ transcription units $\mid s h v$ alleles) or by separating cis-regulatory elements from the transcription units (disk and shv alleles).

\section{disk region}

Northern blot analysis has shown that most of the disk region is not transcribed. Even if the three rare transcripts detected with a probe that extends from 109.5 to 111.0 are transcribed from $d p p$, they cannot encode the major disk functions of the gene, as the $d$-blk deficiency (106-111.4) removes the region that hybridizes to these RNAs but only affects the development of the eye. The genetic properties of disk mutations described in the previous section provide further support for the view that this region does not encode any transcripts that are required for $d p p$ imaginal disk functions. We have sequenced the entire $3.7-\mathrm{kb}$ fragment extending from 109.5 to 113.2 ; it is relatively AT-rich and contains no extended ORFs (data not shown).

Several lines of evidence indicate that the transcripts from the shv and Hin regions are required for the disk functions. Mutations that disrupt all of these transcription units (Hin alleles) are completely amorphic for the disk functions of $d p p$, and proximal shv mutations that break within transcription unit $\mathrm{C}$ also engender disk mutant phenotypes in trans to disk alleles. Mosaic analysis has shown that only the cells that run along the anterior side of the anterior-posterior compartment boundary of the wing disk need to be genotypically $d p p^{+}$ for normal wing development to occur (L.M. Posakony, L.A. Raftery, R.D. St. Johnston and W.M. Gelbart, in prep.). In early third-instar wing disks, the shv-Hin region transcripts are expressed in a band of cells that corresponds to the expected position of the compartment boundary at this stage. These observations directly correlate the location of tissue requiring wild-type disk function with the position of shv-Hin transcript accumulation.

In light of these results, we propose that the disk region consists of a series of cis-regulatory elements that control the imaginal disk expression of the shv and Hin region transcripts. Preliminary data in support of this view are provided by the observation that disk region DNA directs the expression of heterologous promoters along the anterior-posterior compartment boundary in imaginal disks (R.K. Blackman et al., in prep.). According to this model, the disk mutations associated with rearrangements confer their phenotypes by removing cis-regulatory elements from a position adjacent to the shv-Hin transcription units. This model explains the correlation between the severity of a disk mutant phenotype and the proximity of its breakpoint to the Hin region. Breakpoints that fall closer to the Hin region will displace more cis-regulatory information and thus affect more of the $d p p$ expression in imaginal disks. A similar model has been proposed to account for the properties of the bithoraxoid $(b x d)$ alleles of Ultrabithorax (Bender et al. 1985). The small internal deletions in the disk region confer milder phenotypes than breakpoint alleles because they remove only those elements that fall within the deletion, leaving more proximal elements adjacent to the shv-Hin transcription units. The phenotypes of the internal deletions suggest that at least some of the putative cis-regulatory elements are specific to a particular class of imaginal disk. For example, the $d$-blk deficiency only affects the development of the eye, whereas $d$-ho just produces a wing phenotype.

The position of cis-regulatory elements within the disk region is unusual, because the disk region lies $3^{\prime}$ to the $d p p$ transcription units and is separated from them by the two tRNA ${ }^{\text {Tyr }}$ genes. Some of these regulatory elements must lie proximal to $111-112$, the most proximal position of disk-II breakpoints, and some must be located proximal to 116 (on the basis of the comparison of 
the phenotypes of homozygous hb3-blk and disk-II adults). These most distant $3^{\prime}$ cis-regulatory elements are therefore $\geqslant 30 \mathrm{~kb}$ downstream from the nearest $d p p$ promoter.

The larval lethal phenotype elicited by the two mutations falling between the two tRNA ${ }^{\text {Tyr }}$ genes $\mid t 24$ and $t 63$ ) indicates some novel property of this region. Conceivably, these two mutations identify yet another set of $3^{\prime}$ cis-regulatory elements controlling the shv-Hin transcription units. Alternatively, the larval lethal phenotype elicited by these mutations may be a composite of both the loss of all disk region function and the loss of normal tRNA ${ }^{\text {Tyr }}$ activity.

\section{shv region}

The genetic properties of the shv region mutations are similar to those of the disk region. However, because the $5^{\prime}$ exons of several $d p p$ transcripts fall within or beyond the shv region, $s h v$ mutations can affect $d p p$ expression both by removing cis-regulatory elements and by disrupting particular transcription units. The localization of $s h v$ mutations defines a region of $\sim 10 \mathrm{~kb}$ (72.583.7), which contains the $5^{\prime}$ exons of transcripts $C$ and $D$ and the rare embryonic transcripts that hybridize to probes from the 77-79 region. However, this region ends $\sim 12 \mathrm{~kb}$ proximal to the $5^{\prime}$ exon of transcript B. All $s h v$ alleles have been recovered on the basis of their wing venation phenotypes. The failure to recover $s h v$ mutations in the distal portion of this transcription unit suggests that the expression of transcript $B$ is not required for the $d p p$ function in wing venation. Because the most distal shv lesions ( $11, s 6, s 11, s 22, s 20, s 2, s 3$, and $s 7$ ) fall upstream of all of the other $d p p$ transcription units, these mutations probably affect the regulation of RNAs with more proximal promoters. $s 1$ and $s 6$ are both small deletions that only affect the development of the longitudinal wing veins. These deletions overlap, suggesting that a cis-regulatory element that is required for the expression of the $d p p$ product in the wing veins lies in the $72-73$ region.

The larval lethal $s h v$ alleles have been subdivided into two classes on the basis of whether they fully complement the disk alleles or not. The boundary between these two classes falls very close to the $5^{\prime}$ end of transcript C. All of the complementing alleles break $5^{\prime}$ to this transcription unit ( $s 15$ and $s 23$ break within transcript $\mathrm{D}$, which is initiated $\geqslant 25 \mathrm{~kb}$ upstream of the $5^{\prime}$ end of transcript $\mathrm{Cl}$, and all but one of the noncomplementing alleles break within transcript $\mathrm{C}$. The position of this boundary suggests that transcript $C$ is required for the $d p p$ functions in imaginal disks, whereas the transcripts with more distal promoters are not.

\section{Concluding remarks}

The molecular analysis of $d p p$ has allowed us to elaborate a molecular model for the genetic complexity of the locus. Although $d p p$ is required for a variety of developmental events in Drosophila, there is no evidence that alternative splicing produces more than one protein product. Thus, it appears that a single secreted factor can fulfill several developmental functions. This product is encoded by at least five distinct RNAs, each of which has its own promoter and is subject to differing regulatory signals. In this respect, $d p p$ is similar to the Antennapedia and alcohol dehydrogenase genes, in that two promoters give rise to transcripts with the same proteincoding information but different temporal and spatial patterns (Benyajati et al. 1983; Jorgenson and Garber 1987). Most of the genetic complexity of $d p p$ arises from the complicated arrangement of cis-regulatory sequences within the gene (Fig. 5). Some of these regulatory elements must lie at least $30 \mathrm{~kb}$ downstream from the promoter(s) on which they act. Similarly distant regulatory elements have been proposed to exist in the Ultrabithorax gene, defined by $a b x$ mutations that map within an intron, $\sim 40 \mathrm{~kb}$ downstream from the $U b x$ promoter, and by $b x d$ alleles that are localized an equivalent distance upstream (Bender et al. 1985; Hogness et al. 1985; Peifer and Bender 1986).

The variety of phenotypes engendered by disk region mutations leads us to believe that this region contains many separate cis-regulatory elements arranged in tandem. It will be interesting to discover whether these elements operate in combinatorial fashion or whether each element independently specifies a part of the $d p p$ expression pattern in the construction of imaginal tissues. The large distance between some of these regulatory elements and the promoters on which they act suggests that they may have properties in common with vertebrate enhancer elements (Maniatis et al, 1987). Further experiments using P-mediated transformation to insert fragments of the disk region next to heterologous promoters will help to answer these questions.

\section{Experimental procedures}

\section{The cloning of dpp by transposon tagging}

Our molecular analysis of the $d p p$ region began with the generous assistance of E. Strobel and G. Rubin, who provided us with polytene in situ hybridizations of an unstable strain of $d p p$ (called $d 1$ ) probed with a panel of 17 different middle repetitive elements. One of these elements, termed 2177, exhibited hybridization to polytene band $22 \mathrm{~F} 1,2$ (the band containing the $d p p$ gene) in spontaneous revertants of the $d 1$ strain. Subsequently, 2177 proved to be a member of the F element family (Di Nocera et al. 1983). Although the 2177 insertion proved not to be responsible for the $d 1$ mutation, a clone from $22 \mathrm{~F} 1,2$ was obtained by probing a genomic $d 1$ revertant library with this element, following the general methodology for transposon tagging (Bingham et al. 1981).

An EcoRI partial genomic library containing DNA fragments from a strain containing an unstable revertant of $d 1$, called $+d 1 R$, was constructed in the phage $\lambda$ vector Charon 4 (prepared by Vivian Irish). (The $d 1$ revertant derivative also contains 2177 homologous sequences in $22 \mathrm{Fl}, 2$.) Over 200 phage bearing 2177 homologous DNAs were plaque-purified. They were screened for the presence of $22 \mathrm{~F} 1,2$ sequences by in situ hybridization to polytene chromosomes of Drosophila simulans, a sibling species not containing 2177 elements in the vicinity of $22 \mathrm{~F} 1,2$. 


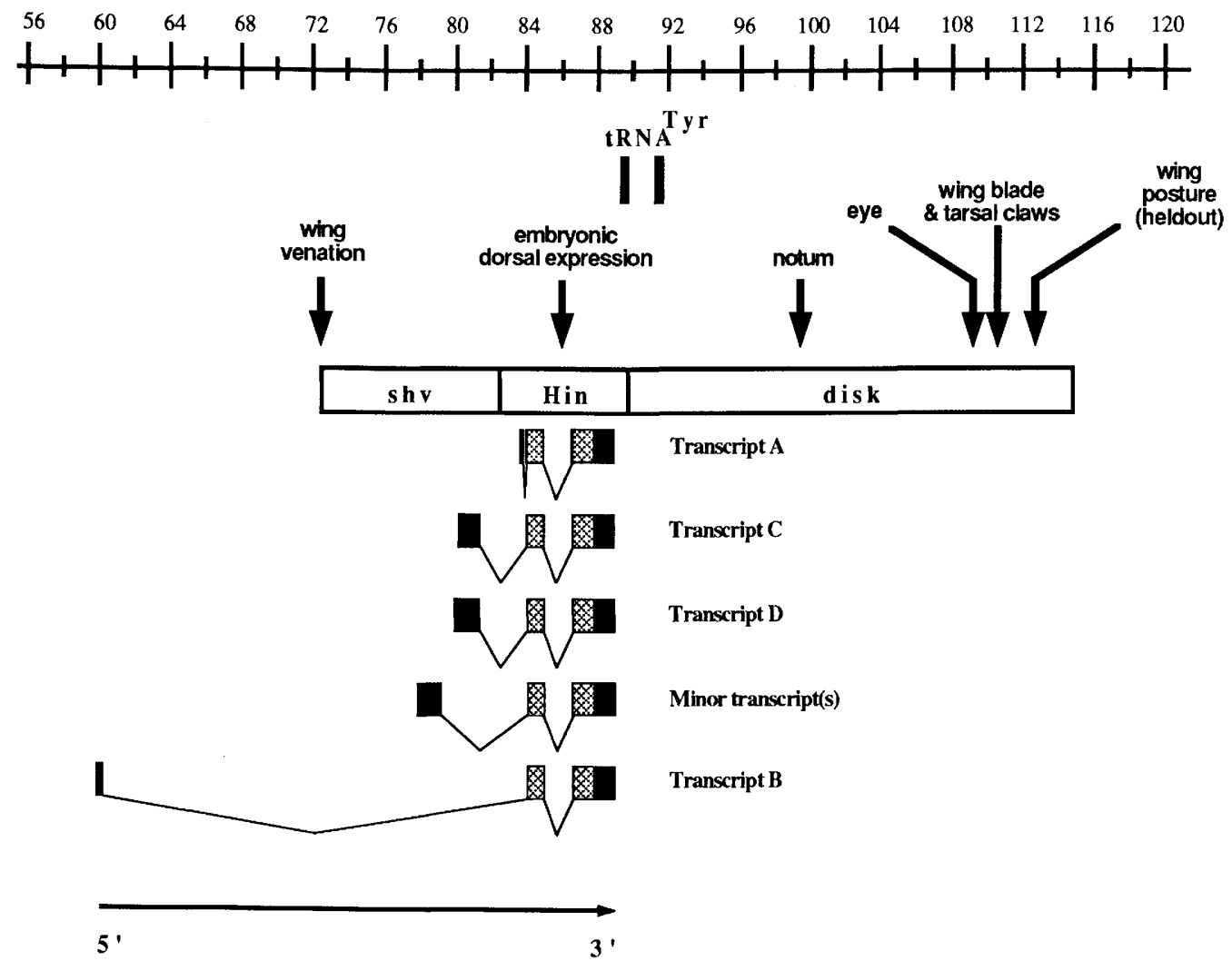

Figure 5. Summary map of $d p p$ gene. The location of the three major $d p p$ domains is depicted below the scale (in kb). Above the scale, the approximate locations of cis-regulatory elements controlling discrete events in the contribution $d p p$ to normal embryonic and adult development are depicted. Many other cis-regulatory elements controlling temporal, spatial, and quantitative aspects of $d p p$ expression are present within the gene but have not yet been localized. The locations of exons for five alternative $d p p$ transcripts are represented at bottom. For details of this transcriptional map, see Fig. 3.

\section{Chromosomal walk to dpp}

Unique sequence DNA from $\lambda$-204 hybridized in situ only to polytene bands $22 \mathrm{~F} 1,2$. This fragment was used to probe phage clones from a Canton-S library (Maniatis et al. 1978) to initiate a chromosomal walk (Bender et al. 1983). After walking $\sim 20 \mathrm{~kb}$ in either direction from the $\mathrm{F}$ element, the orientation of the walk was determined by in situ hybridization of probes from either end of the walk to polytene chromosomes bearing Hin32, a deletion removing all of $22 \mathrm{~F}$ and exhibiting a null $d p p$ phenotype. Probes from the distal end of the walk hybridized to the deletion-bearing chromosome, whereas probes from the proximal end did not. The remainder of the walk was carried out only in the proximal direction. Phage clones spanning $140 \mathrm{~kb}$ of genomic DNA from the $22 \mathrm{Fl}, 2$ region were isolated. The distal end of the walk was designated map position 0 , and the proximal end map position was 140 (in kb).

\section{Genomic libraries}

For the original chromosome walk through the $d p p$ gene, a genomic library of randomly sheared Drosophila Canton-S DNA cloned into Charon-4A (Maniatis et al. 1978) was employed. For several cytologically normal alleles containing restriction map

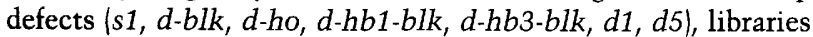
of mutant DNA were prepared in EMBL3 (Maniatis et al. 1982).

Mutant localization by restriction mapping and chromosomal in situ hybridization analyses

Mutations were localized on the molecular map by two dif- ferent strategies, depending on the cytogenetic properties of the $d p p$ alleles. Most extant $d p p$ mutations are associated with gross chromosomal rearrangements (inversions and translocations) with breakpoints in $22 \mathrm{~F} 1,2$. The molecular positions of 48 such $d p p$ rearrangements were initially determined by in situ hybridization of subcloned DNA fragments from the chromosomal walk to polytene chromosomes of $d p p /+$ heterozygotes. For mutations located near the boundaries separating lesions of different phenotypic classes, more precise localization was obtained by whole genome Southern blot analysis. Note that rearrangements juxtaposing $d p p$ to heterochromatin have been excluded from this molecular analysis, because these mutations might affect $d p p$ functions from a distance as a result of position effect variegation.

Twenty-one cytologically normal alleles were analyzed by whole genome Southern blotting. In 6 of the 12 alleles exhibiting molecular alterations, further information was obtained from the restriction maps of cloned mutant DNA. Nine cytologically normal lesions exhibited no restriction map abnormalities. The lesions in these mutants are presumably either true point mutations or rearrangements of $<0.2 \mathrm{~kb}$.

\section{RNA preparations and Northern blots}

Total RNA was isolated from 12 developmental stages using the hot phenol method, and passed once over a poly(U)-Sepharose column to prepare poly(A) ${ }^{+}$RNA (Shapiro and Schimke 1975). Two micrograms of poly(A) ${ }^{+}$RNA were run in each lane on $1 \%$ formaldehyde agarose gels, as described by Maniatis et 
al. (1982) and transferred to nylon membranes. The shv and Hin regions (68-93, Fig. 1) were subcloned into M13 vectors, and single-stranded ${ }^{32} \mathrm{P}$-labeled DNA probes were synthesized from M13 templates, following a procedure adapted from Akam (1983). The disk region (93-119, Fig. 1) was screened first with [32P]UTP-labeled RNA from an SP6 vector (Promega). Seven clones hybridized to one or more transcripts. These regions were recloned into an M13 vector, and single-stranded DNA probes were prepared as above. Only two regions hybridized to the DNA probes (see Results). The sizes of the $d p p$ RNAs were determined by running ${ }^{35}$ S-labeled SP6 transcripts of known molecular weights as size standards on one lane of each gel. Several of the Northern blots were rehybridized with a nicktranslated $r 49$ probe to control for the loading of RNA in each lane. The $r p 49$ gene encodes a ribosomal protein and is expressed at approximately the same level throughout development (Al-Atia et al. 1985).

\section{cDNA libraries}

$d p p$ cDNA clones have been recovered from the following libraries: 0- to 5-hr embryonic (a gift of $\mathrm{M}$. Goldschmidt-Clermont, R. Saint, and D. Hogness), 3- to 12-hr embryonic (Poole et al. 1985), 4- to 8-hr embryonic (Brown and Kafatos 1988), 8to 12-hr embryonic (Brown and Kafatos 1988) and 5.5- to 7.5day-old pupae (Poole et al. 1985). Each of these libraries was also screened against the entire disk region, but no cDNA clones were recovered.

\section{Revised dpp nomenclature}

As described in this report, we feel that it is not appropriate to refer to a "decapentaplegic gene complex," but rather to treat $d p p$ as a single gene with complex allelic interactions. Our genetic nomenclature has been revised accordingly. In our new nomenclature, all decapentaplegic mutations are referred to as $d p p$ alleles. $d p p$ mutations are divided into three major subgroups, that are now designated Hin, shv and disk (formerly $H i n-d, s h v$, and $d p p$, respectively). Mutations globally affecting $d p p$ functions are described as $d p p^{H i n x}$ (where $\mathrm{x}$ is the allele number|, for those alleles that have a dominant haplolethal phenotype or $d p p^{h i n-x}$ for those alleles that are recessive lethal. Two major categories of mutations affecting only a subset of $d p p$ functions have been identified. Some of these mutations have been recovered as alleles of the pre-existing mutation called $(s h v)$; these mutations were formerly designated $s h v^{s x}$ but are now referred to as $d p p^{s x}$. Others elicit phenotypic effects restricted to the imaginal disks; these disk mutations, formerly $d p p^{x}$, are now termed $d p p^{d x}$ (where $x$ begins with a number) or $d p p^{d-y}$, where $y$ begins with a letter. Descriptions of the properties of these mutations are presented in Results.

\section{Previously unreported dpp alleles}

Nine $d p p$ mutations are reported here for the first time. Three were $\gamma$-irradiation-induced by L. Posakony (1987): $d 60$ [In(2L) 21E; 22F], Hin61 (cytologically normal), and $t 63[\operatorname{In}(2 \mathrm{~L}) 22 \mathrm{~F}$; $39 \mathrm{C}-\mathrm{D}]$. Six were $\gamma$-irradiation-induced and recovered as a byproduct of screens reported by Segal and Gelbart (1985): d66 $[\mathrm{Dp}(3 ; 2) 78 \mathrm{~F} ; 80 \mathrm{~F}$ into $\operatorname{In}(2 \mathrm{~L}) 21 \mathrm{E} ; 22 \mathrm{~F}], d 68$ [In(2LR) 22F; 23D; $51 \mathrm{D}], d 70[\mathrm{Tp}(2 ; 2) 21 \mathrm{D} ; 22 \mathrm{~F}$ into $21 \mathrm{~A}], d 72[\mathrm{Tp}(2 ; 3) 22 \mathrm{~F} ; 34 \mathrm{~B}$ into $81 \mathrm{~F}], d 74$ [ $\mathrm{Tp}(2 ; 2) 26 \mathrm{~A} ; 29 \mathrm{D}-\mathrm{E}$ into $22 \mathrm{~F}$ ].

\section{Acknowledgments}

We acknowledge the excellent technical assistance of Ruth Ca- tavalo, Marcy Taylor, Margaret de Cuevas, Lee Kerkhof, and Pablo Figueroa. We are grateful to Ed Strobel and Gerald Rubin for their considerable help in identifying the mobile element insertion that allowed us to begin a chromosome walk through $22 \mathrm{~F} 1,2$. This work was supported by a grant from the National Institutes of Health (NIH) to W.M.G. During the course of this work, F.M.H. and R.W.P. were postdoctoral fellows of the Massachusetts Medical Foundation, R.K.B., R.W.P, and H.A.I. were NIH postdoctoral fellows, D.S. was an American Cancer Society postdoctoral fellow, and R.G. was an NIH predoctoral trainee.

The publication costs of this article were defrayed in part by payment of page charges. This article must therefore be hereby marked "advertisement" in accordance with 18 USC section 1734 solely to indicate this fact.

\section{References}

Akam, M.E. 1983. The location of $U b x$ transcripts in Drosophila tissue sections. EMBO /. 2: 2075-2084.

Al-Atia, G.R., P. Fruscoloni, and M. Jacobs-Lorena. 1985. Translational regulation of mRNAs for ribosomal proteins during early Drosophila development. Biochemistry 24: 5798-5803.

Anderson, K.V. 1987. Dorsal-ventral embryonic pattern genes of Drosophila. Trends Genet. 3: 91-97.

Baker, N. 1988. Transcription of the segment polarity gene wingless in the imaginal discs of Drosophila, and the phenotype of a pupal lethal wingless mutation. Development 102: 489-497.

Bender, W., P. Spierer, and D. Hogness. 1983. Chromosomal walking and jumping to isolate DNA from the Ace and rosy loci and the bithorax complex in Drosophila melanogaster. I. Mol. Biol. 168: 17-33.

Bender, W., B. Weiffenbach, F. Karch, and M. Peifer. 1985. Domains of cis-interaction in the Bithorax complex. Cold Spring Harbor Symp. Quant. Biol. 50: 173-180.

Benyajati, C., N. Spoerel, H. Haymerle, and M. Ashburner. 1983. The messenger RNA for alcohol dehydrogenase in Drosophila melanogaster differs in its $5^{\prime}$ end in different developmental stages. Cell 33: 125-133.

Bingham, P.M., R. Levis, and G.M. Rubin. 1981. Cloning of DNA sequences from the white gene of $D$. melanogaster by a novel and general method. Cell 25: 693-704.

Blackman, R.K., R. Grimaila, M.M.D. Koehler, and W.M. Gelbart. 1987. Mobilization of hobo elements residing within the decapentaplegic gene complex: Suggestion of a new hybrid dysgenesis system in Drosophila melanogaster. Cell 49: 497-505.

Boulay, J.L., C. Dennefeld, and A. Alberga. 1987. The Drosophila developmental gene snail encodes a protein with nucleic acid binding fingers. Nature 330: 395-398.

Brown, N.H. and F.C. Kafatos. 1988. Functional cDNA libraries from Drosophila embryos. J. Mol. Biol. 203: 425-437.

Bryant, P.J. 1988. Localized cell death caused by mutations in a Drosophila gene coding for a transforming growth factor $-\beta$ homolog. Dev. Biol. 128: 386-395.

Carroll, S.B. and J.S. White. 1989. The role of the hairy gene during Drosophila morphogenesis: Stripes in imaginal discs. Genes Dev. 3: 905-916.

Cate, R.L., R.J. Mattalliano, C. Hession, R. Tizard, N.M. Farber, A. Cheung, E.G. Ninfa, A.Z. Frey, D.J. Gash, E.P. Chow, R.A. Fisher, J.M. Bertonis, G. Torres, B.P. Wallner, K.L. Ramachandran, R.C. Ragin, T.F. Manganaro, D.T. MacLaughlin, and P.T. Donahoe. 1986. Isolation of the bovine and human genes for Müllerian inhibiting substance 
and expression of the human gene in animal cells. Cell 45: 685-698.

Derynk, R., J.A. Jarrett, E.Y. Chen, D.H. Eaton, J.R. Bell, R.K. Assoian, A.B. Roberts, M.B. Sporn, and D.V. Goeddel. 1985. Human transforming growth factor- $\beta$ complementary DNA sequence and expression in normal and transformed cells. Nature 316: 701-705.

Di Nocera, P.P., M.E. Digan, and I.B. Dawid. 1983. A family of oligo-adenylate-terminated transposable elements in Drosophila melanogaster. J. Mol. Biol. 168: 715-727.

Doyle, H.J., K. Harding, T. Hoey, and M. Levine. 1986. Transcripts encoded by a homoeo box gene are restricted to dorsal tissue of Drosophila embryos. Nature 323: 76-79.

Gelbart, W.M., V.F. Irish, R.D. St. Johnston, F.M. Hoffmann, R. Blackman, D. Segal, L.M. Posakony, and R. Grimaila. 1985. The decapentaplegic gene complex in Drosophila melanogaster. Cold Spring Harbor Symp. Quant. Biol. 50: 119125.

Hogness, D.S., H.D. Lipshitz, P.A. Beachy, D.A. Peattie, R.B. Saint, M. Goldschmidt-Clermont, P.J. Harte, E.R. Gavis, and S.L. Helfand. 1985. Regulation and products of the Ubx domain of the Bithorax complex. Cold Spring Harbor Symp. Quant. Biol. 50: 181-194.

Hoffmann, F.M. and W. Goodman. 1987. Identification in transgenic animals of the Drosophila decapentaplegic sequences required for normal embryonic pattern formation. Genes Dev. 1: 615-625.

Irish, V.F. and W.M. Gelbart. 1987. The haplo-insufficient region of the decapentaplegic gene is required for dorsal-ventral patterning of the Drosophila embryo. Genes Dev. 1: $868-879$.

Jorgenson, E.M. and R.L. Garber. 1987. Function and misfunction of the two promoters of the Drosophila Antennapedia gene. Genes Dev. 1: 544-555.

Jürgens, G., E. Wieschaus, C. Nüsslein-Volhard, and H. Kluding. 1984. Mutations affecting the pattern of the larval cuticle in Drosophila melanogaster. II. Zygotic loci on the third chromosome. Wilhelm Roux's Archiv. Dev. Biol. 193: $283-295$.

Maniatis, T., R.C. Hardison, E. Lacy, J. Lauer, C. O'Connell, D. Quon, G.K. Sim, and A. Efstratiadis. 1978. The isolation of structural genes from libraries of eukaryotic DNA. Cell 15: $687-701$.

Maniatis, T., E.F. Fritsch, and J. Sambrook. 1982. Molecular cloning: A laboratory manual. Cold Spring Harbor Laboratory, Cold Spring Harbor, New York.

Maniatis, T., S. Goodbourn, and J. Fischer. 1987. Regulation of inducible and tissue-specific gene expression. Science 236: $1237-1244$.

Mason, A.J., J.S. Hayflick, N. Ling, F. Esch, N. Ueno, S.-Y. Ying, R. Guillemin, H. Naill, and R.H. Seeburg. 1985. Complementary DNA sequences of ovarian follicular fluid inhibin show precursor structure and homology with transforming growth factor- $\beta$. Nature 318: 659-663.

Murre, C., P. Schonleber-McCaw, and D. Baltimore. 1989. A new DNA-binding and dimerization motif in immunoglobulin enhancer binding, daughterless, MyoD and myc proteins. Cell 56: 777-783.

Nüsslein-Volhard, C., E. Wieschaus, and H. Kluding. 1984. Mutations affecting the pattern of the larval cuticle in Drosophila melanogaster. I. Zygotic loci on the second chromosome. Wilhelm Roux's Archiv. Dev. Biol. 193: 267-282.

Padgett, R.W., R. Daniel St. Johnston, and W.M. Gelbart. 1987. A transcript from a Drosophila pattern gene predicts a protein homologous to the transforming growth factor- $\beta$ gene family. Nature 325: 81-84.
Peifer, M. and W. Bender. 1986. The anterobithorax and bithorax mutations of the bithorax complex. EMBO $/$. 1: 892-898.

Poole, S., L.M. Kauvar, B. Drees, and T. Kornberg. 1985. The engrailed locus of Drosophila: Structural analysis of an embryonic transcript. Cell 40: $37-43$.

Posakony, L.M. 1987. The role of the DPP-C in the development of the imaginal discs in Drosophila melanogaster. Ph.D. dissertation, Harvard University, Cambridge.

Roth, S., D. Stein, and C. Nüsslein-Volhard. 1989. A gradient of nuclear localization of the dorsal protein determines dorsoventral pattern in the Drosophila embryo. Cell 59: 11891202.

Rushlow, C.A, M. Frasch, H. Doyle, and M. Levine. 1987. Maternal regulation of zerknüllt: A homoeobox gene controlling differentiation of dorsal tissues in Drosophila. Nature 330: $583-586$.

Rushlow, C.A., K. Han, J.L. Manley, and M. Levine. 1989. The graded distribution of the dorsal morphogen is initiated by selective nuclear transport in Drosophila. Cell 59: 11651177.

St. Johnston, R.D. 1988. The structure and expression of the decapentaplegic gene in Drosophila melanogaster. Ph.D. dissertation, Harvard University, Cambridge.

St. Johnston, R.D. and W.M. Gelbart. 1987. Decapentaplegic transcripts are localized along the dorsal-ventral axis of the Drosophila embryo. EMBO I. 6: 2785-2791.

Segal, D. and W.M. Gelbart. 1985. Shortvein, a new component of the decapentaplegic gene complex in Drosophila melanogaster. Genetics 109: 119-143.

Shapiro, D.J. and R.T. Schimke. 1975. Immunochemical isolation and characterization of ovalbumin messenger ribonucleic acid. J. Biol. Chem. 250: 1759-1764.

Spencer, F.A., F.M. Hoffmann, and W.M. Gelbart. 1982. Decapentaplegic: A gene complex affecting morphogenesis in Drosophila melanogaster. Cell 28: 451-461.

Steward, R. 1989. Relocalization of the dorsal protein from the cytoplasm to the nucleus correlates with its function. Cell 59: $1179-1188$.

Thisse, B., C. Stoetzel, M. El Messal, and F. Perrin-Schmitt. 1987. Genes of the Drosophila maternal dorsal group control the specific expression of the zygotic gene twist in presumptive mesodermal cells. Genes Dev. 1: 709-715.

Thisse, B., C. Stoetzel, C. Gorostiza-Thisse, and F. PerrinSchmitt. 1988. Sequence of the $t$ wist gene and nuclear localization of its protein in endomesodermal cells of early Drosophila embryos. EMBO J. 7: 2175-2183.

Wakimoto, B.T., F.R. Turner, and T.C. Kaufman 1984. Effects in embryogenesis in mutants associated with the Antennapedia gene complex of Drosophila melanogaster. Dev. Biol. 102: $147-172$.

Weeks, D.L. and D.A. Melton. 1987. A maternal mRNA localized to the vegetal hemisphere in Xenopus eggs codes for a growth factor related to TGF- $\beta$. Cell 51: 861-867.

Wozney, J.M., V. Rosen, A.J. Celeste, L.M. Mitsock, M.J. Whitters, R.K. Kriz, R.M. Hewick, and E.A. Wang. 1988. Molecular cloning of novel regulators of bone formation. Science 242: 1528-1534.

Zusman, S.B. and E. Wieschaus. 1985. Requirements for zygotic gene activity during gastrulation in Drosophila melanogaster. Dev. Biol. 111: 359-371.

Zusman, S.B., D. Sweeton, and E.F. Wieschaus. 1988. short gastrulation, a mutation causing delays in stage-specific cell shape changes during gastrulation in Drosophila melanogaster. Dev. Biol. 129: 417-427. 


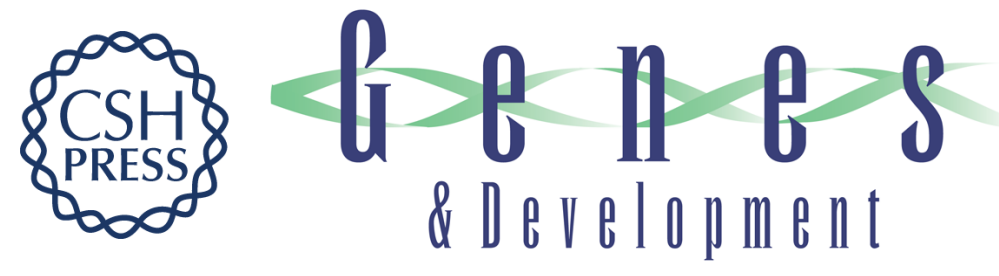

\section{Molecular organization of the decapentaplegic gene in Drosophila melanogaster.}

R D St Johnston, F M Hoffmann, R K Blackman, et al.

Genes Dev. 1990, 4:

Access the most recent version at doi:10.1101/gad.4.7.1114

References This article cites 46 articles, 13 of which can be accessed free at: http://genesdev.cshlp.org/content/4/7/1114.full.html\#ref-list-1

License

Email Alerting

Service

Receive free email alerts when new articles cite this article - sign up in the box at the top right corner of the article or click here.

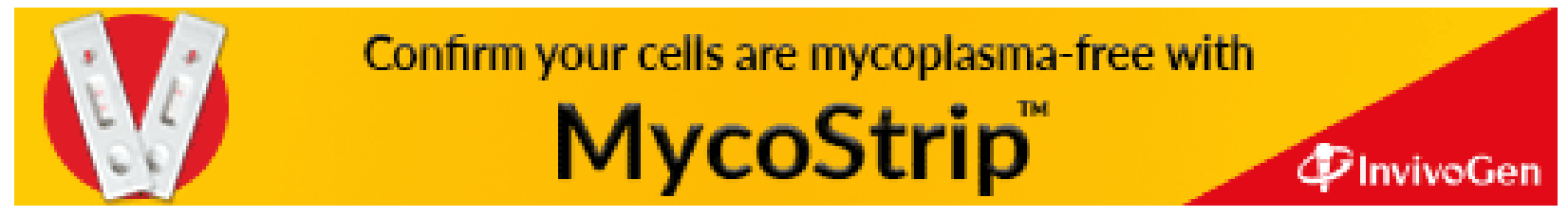

